\title{
ON THE INEQUALITY OF KSHIRSAGAR
}

\author{
Ken-ichi Koike \\ Institute of Mathematics, University of Tsukuba, \\ Tsukuba, Ibaraki 305-8571, Japan
}

Key words and Phrases: Hammersley-Chapman-Robbins bound, Cramér-Rao bound, Bhattacharyya bound, Kshirsagar bound, unbiased estimators, complete sufficient statistics.

AMS 1991 subject classification. Primary 62F10.

\begin{abstract}
A lower bound for the variance of unbiased estimators which is due to Kshirsagar is studied. The bound is free from regularity assumptions. It is also shown that the bound is an improvement of Bhattacharyya bound in regular case.
\end{abstract}

\section{INTRODUCTION}

Let $X$ be a random variable according to the density function $f(x, \theta)(\theta \in$ $\left.\Theta \subset \mathbb{R}^{1}\right)$ with respect to $\sigma$-finite measure $\mu$. The support $S(\theta)$ of the density functions is defined by $S(\theta):=\{x: f(x, \theta)>0\}$.

Let $g$ be a differentiable function on $\Theta$, not identically constant. Let $\hat{g}(X)$ be an unbiased estimator of $g(\theta)$ with $E_{\theta}\left\{\hat{g}(X)^{2}\right\}<\infty$. 
The Bhattacharyya bound states that, under some regularity conditions $R_{k}$ (see Fend (1959)),

$$
\begin{aligned}
\operatorname{Var}_{\theta}\{\hat{g}(X)\} & \geq\left(g^{(1)}(\theta), \ldots, g^{(k)}(\theta)\right) W^{-1}(\theta)\left(g^{(1)}(\theta), \ldots, g^{(k)}(\theta)\right)^{\prime} \\
& =: B_{k}(\theta), \quad \text { say }
\end{aligned}
$$

where $g^{(i)}(\theta)=\frac{d^{i} g(\theta)}{d \theta^{i}}$ and $W(\theta):=\left\{w_{i j}(\theta)\right\}_{i, j=1, \ldots, k}$ with

$$
w_{i j}(\theta):=E_{\theta}\left\{\frac{\frac{\partial^{i} f(X, \theta)}{\partial \theta^{i}} \frac{\partial^{j} f(X, \theta)}{\partial \theta^{j}}}{f^{2}(X, \theta)}\right\} \quad(i, j=1, \ldots, k) .
$$

It is well known that $B_{1}(\theta)$ is identical to the Cramér-Rao bound and $B_{k+1}(\theta) \geq$ $B_{k}(\theta)(k \geq 1)$ for $\theta \in \Theta$.

The Hammersley-Chapman-Robbins bound states that

$$
\operatorname{Var}_{\theta}\{\hat{g}(X)\} \geq \sup _{\phi} \frac{\{g(\phi)-g(\theta)\}^{2}}{E_{\theta}\left\{\frac{f(X, \phi)-f(X, \theta)}{f(X, \theta)}\right\}^{2}}=: H(\theta), \quad \text { say, }
$$

where the supremum is taken over the set of all $\phi \in \Theta$ satisfying

$$
g(\phi) \neq g(\theta) \text { and } S(\phi) \subset S(\theta) .
$$

This bound does not need the assumptions of the common support and the existence of the derivative of the density function. It is also well known that $H(\theta) \geq B_{1}(\theta)$ (see Chapman and Robbins (1951) and Sen and Ghosh (1976)).

Recently, Kshirsagar (2000) gave an extension of Hammersley-ChapmanRobbins bound in the same manner as Bhattacharyya bound. Let

$$
\psi_{r}:=\frac{f\left(x, \phi_{r}\right)-f(x, \theta)}{f(x, \theta)} \quad(r=1,2, \ldots, k) .
$$

The Kshirsagar bound states that

$$
\operatorname{Var}_{\theta}\{\hat{g}(X)\} \geq \sup _{\phi} \mathbf{w} \Sigma^{-1} \mathbf{w}^{\prime}=: K_{k}(\theta), \quad \text { say }
$$

where $\mathbf{w}=\mathbf{w}\left(\theta, \phi_{1}, \ldots, \phi_{k}\right):=\left(g\left(\phi_{1}\right)-g(\theta), \ldots, g\left(\phi_{k}\right)-g(\theta)\right)$, $\Sigma=\Sigma\left(\theta, \phi_{1}, \ldots, \phi_{k}\right)=\left\{\sigma_{i j}\right\}_{i, j=1, \ldots, k}$ with $\sigma_{i j}=\operatorname{cov}_{\theta}\left(\psi_{i}, \psi_{j}\right)(i, j=1, \ldots, k)$ and the supremum is taken over the set of all $\phi_{i} \in \Theta(i=1, \ldots, k)$ satisfying

$$
S\left(\phi_{k}\right) \subset S\left(\phi_{k-1}\right) \subset \cdots \subset S\left(\phi_{1}\right) \subset S(\theta)
$$


The purpose of the paper is to show another extension of HammersleyChapman-Robbins bound in the same manner as Kshirsagar (2000) and some relations with usual Bhattacharyya bound by using the bound.

\section{ANOTHER LOWER BOUND OF THE VARIANCE FOR UNBIASED ESTIMATORS}

Let $X$ be a random variable with the density function $f(x, \theta)(\theta \in \Theta)$ with respect to a $\sigma$-finite measure $\mu$, where $\Theta$ is a subset of $\mathbb{R}^{1}$. Consider the problem of unbiased estimation for a given real-valued nonconstant function $g(\theta)$. At first, we have the following lemma.

Lemma 1. If $g(\theta)$ is $k$ times continuously differentiable, then it holds

$$
G_{i}=G_{i}(\theta, \delta):=\left(\frac{-1}{\delta}\right)^{i} \sum_{l=0}^{i}\left(\begin{array}{l}
i \\
l
\end{array}\right)(-1)^{l} g(\theta+l \delta) \rightarrow g^{(i)}(\theta) \quad(i=1, \ldots, k)
$$

as $\delta \rightarrow 0$, where $g^{(i)}$ is the $i$-th derivative of $g(\theta)$ at $\theta(i=1, \ldots, k)$.

The proof is omitted since it is an easy consequence of L'Hospitals theorem.

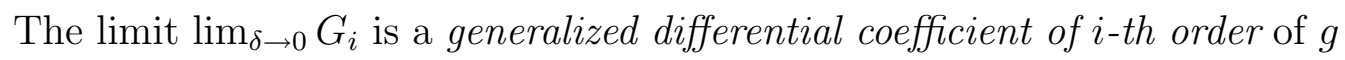
at $\theta$. For example, take $g(\theta)=|\theta|$. Since it is not differentiable at $\theta=0$, we cannot compute the 2 nd differential coefficient of $g(\theta)$ at $\theta=0$. But, on the other hand, $G_{2}=\{g(\theta+2 \delta)-2 g(\theta+\delta)+g(\theta)\}=0$ and hence $\lim _{\delta \rightarrow 0} G_{2}=0$.

Let $S(\theta)$ be the support of $f(x, \theta)$. Assume that we can take $\theta+i \delta \in \Theta$ $(i=1, \ldots, k)$ such that $S(\theta) \supset S(\theta+i \delta)(i=1, \ldots, k)$. Define

$$
\Psi_{i}=\Psi_{i}(x, \theta, \delta):=\left(\frac{-1}{\delta}\right)^{i} \sum_{l=0}^{i}\left(\begin{array}{l}
i \\
l
\end{array}\right)(-1)^{l} \frac{f(x, \theta+l \delta)}{f(x, \theta)} \quad(i=1, \ldots, k) .
$$

Let

$$
V=V(\theta, \delta)=\left\{v_{i j}(\theta, \delta)\right\}
$$

where

$$
v_{i j}(\theta, \delta)=E_{\theta}\left(\Psi_{i} \Psi_{j}\right) \quad(i, j=1, \ldots, k) .
$$


Then we have an extension of the Hammersley-Chapman-Robbins bound as follows.

Theorem 1. Let $\hat{g}(X)$ be an unbiased estimator of $g(\theta)$. Then it holds

$$
\operatorname{Var}_{\theta}\{\hat{g}(X)\} \geq \sup _{\delta \in \Delta} \mathbf{g} V^{-1} \mathbf{g}^{\prime}=: D_{k}(\theta), \quad \text { say }
$$

where $\mathbf{g}=\mathbf{g}(\theta, \delta):=\left(G_{1}, \ldots, G_{k}\right)$ and $\Delta=\{\delta: S(\theta) \supset S(\theta+i \delta) \quad(i=$ $1, \ldots, k),|V(\theta, \delta)| \neq 0\}$. If $\Delta=\emptyset$, set the right-hand side 0 by the usual convention.

Proof. Without loss of generality, we may assume $\Delta \neq \emptyset$. Fix $\delta \in \Delta$. Since $S(\theta) \supset S(\theta+i \delta)(i=1, \ldots, k)$, for $i=1, \ldots, k$,

$$
\begin{aligned}
E_{\theta}\left\{\Psi_{i}(X, \theta, \delta)\right\} & =\left(\frac{-1}{\delta}\right)^{i} \sum_{l=0}^{i}\left(\begin{array}{l}
i \\
l
\end{array}\right)(-1)^{l} E_{\theta}\left\{\frac{f(X, \theta+l \delta)}{f(X, \theta)}\right\} \\
& =\left(\frac{-1}{\delta}\right)^{i} \sum_{l=0}^{i}\left(\begin{array}{l}
i \\
l
\end{array}\right)(-1)^{l} \int_{S(\theta)} f(x, \theta+l \delta) d \mu \\
& =\left(\frac{-1}{\delta}\right)^{i} \sum_{l=0}^{i}\left(\begin{array}{l}
i \\
l
\end{array}\right)(-1)^{l} \\
& =\left(\frac{-1}{\delta}\right)^{i}(1-1)^{i}=0 .
\end{aligned}
$$

Hence, since $\hat{g}(X)$ is an unbiased estimator of $g(\theta)$, we have

$$
\begin{aligned}
\operatorname{Cov}_{\theta}\left(\hat{g}(X), \Psi_{i}(X, \theta, \Delta)\right) & =E_{\theta}\left\{\hat{g}(X) \Psi_{i}(X, \theta, \Delta)\right\} \\
& =\left(\frac{-1}{\delta}\right)^{i} \sum_{l=0}^{i}\left(\begin{array}{l}
i \\
l
\end{array}\right)(-1)^{l} \int_{S(\theta)} \hat{g}(x) f(x, \theta+l \theta) d \mu \\
& =\left(\frac{-1}{\delta}\right)^{i} \sum_{l=0}^{i}\left(\begin{array}{l}
i \\
l
\end{array}\right)(-1)^{l} g(\theta+l \delta) \\
& =G_{i} .
\end{aligned}
$$

Considering the covariance matrix $U$ of the random vector $\left(\hat{g}(X), \Psi_{1}(X, \theta, \delta), \ldots, \Psi_{k}(X, \theta, \delta)\right)$, we can show that $U$ is a symmetric matrix 
given by

$$
U=\left(\begin{array}{cccc}
\operatorname{Var}_{\theta}\{\hat{g}(X)\} & G_{1} & \cdots & G_{k} \\
G_{1} & V_{11}(\theta, \delta) & \cdots & V_{1 k}(\theta, \delta) \\
\vdots & \vdots & \ddots & \vdots \\
G_{k} & V_{k 1}(\theta, \delta) & \cdots & V_{k k}(\theta, \delta) .
\end{array}\right)
$$

Since $U$ is nonnegative definite and $|V|>0$, it follows that

$$
|U|=|V|\left|\operatorname{Var}_{\theta}\{\hat{g}(X)\}-\mathbf{g} V^{-1} \mathbf{g}^{\prime}\right|
$$

And then, we have

$$
\operatorname{Var}_{\theta}\{\hat{g}(X)\} \geq \mathbf{g} V^{-1} \mathbf{g}^{\prime}
$$

Taking the supremum with respect to $\delta$, we obtain the desired inequality.

Remark. For $k=1$, the bound (2.3) is identical to (1.2) and (1.4).

A relation between the bounds (1.4) and (2.3) is given as follows.

Theorem 2. If $S(\theta+i \delta) \subset S(\theta)$ for some $\delta \neq 0(i=1, \ldots, k)$, it holds

$$
\begin{aligned}
& \mathbf{w}(\theta, \theta+\delta, \ldots, \theta+k \delta)\{\Sigma(\theta, \theta+\delta, \ldots, \theta+k \delta)\}^{-1} \mathbf{w}(\theta, \theta+\delta, \ldots, \theta+k \delta)^{\prime} \\
= & \mathbf{g}(\theta, \delta)\{V(\theta, \delta)\}^{-1} \mathbf{g}(\theta, \delta)^{\prime}
\end{aligned}
$$

Proof. Putting $\phi_{i}=\theta+i \delta(i=1, \ldots, k, \delta \neq 0)$ in (1.3), we will show $\mathbf{g}(\theta, \delta) V^{-1} \mathbf{g}(\theta, \delta)^{\prime}=\mathbf{w} \Sigma^{-1} \mathbf{w}^{\prime}$. We see from the definition of $G_{i}$ that, for $i=$ $1, \ldots, k$,

$G_{i}=\left(\frac{-1}{\delta}\right)^{i} \sum_{l=0}^{i}\left(\begin{array}{l}i \\ l\end{array}\right)(-1)^{l} g(\theta+l \delta)=\left(\frac{-1}{\delta}\right)^{i} \sum_{l=1}^{i}\left(\begin{array}{l}i \\ l\end{array}\right)(-1)^{l}\{g(\theta+l \delta)-g(\theta)\}$.

Then, since

$$
\begin{aligned}
& \mathbf{g}=\left(G_{1}, \ldots, G_{k}\right) \\
&=\left(\left(\frac{-1}{\delta}\right)^{1} \sum_{l=1}^{1}\left(\begin{array}{l}
1 \\
l
\end{array}\right)(-1)^{l}\{g(\theta+l \delta)-g(\theta)\}, \ldots,\right. \\
&\left.\left(\frac{-1}{\delta}\right)^{k} \sum_{l=1}^{k}\left(\begin{array}{l}
k \\
l
\end{array}\right)(-1)^{l}\{g(\theta+l \delta)-g(\theta)\}\right)
\end{aligned}
$$




$$
\begin{aligned}
& =(g(\theta+\delta)-g(\theta), \ldots, g(\theta+k \delta)-g(\theta))
\end{aligned}
$$

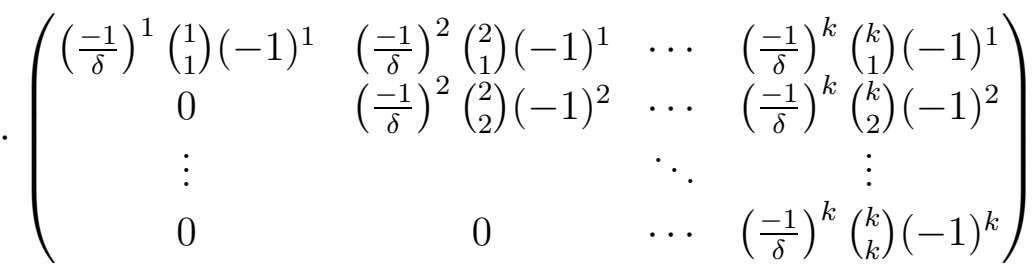

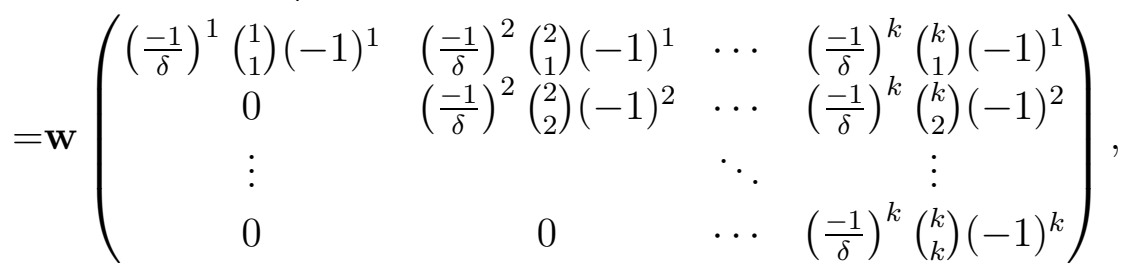

it holds

$$
\begin{aligned}
\mathbf{g} V^{-1} \mathbf{g}^{\prime} & =\mathbf{w} F V^{-1} F^{\prime} \mathbf{w}^{\prime} \\
& =\mathbf{w}\left(\left(F^{\prime}\right)^{-1} V F^{-1}\right)^{-1} \mathbf{w}^{\prime}
\end{aligned}
$$

where $F=\left\{f_{i j}\right\}_{i, j=1, \ldots, k}$ is a nonsingular matrix with

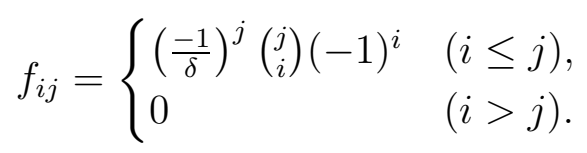

Thus, to prove the theorem, it suffices to show that $\left(F^{\prime}\right)^{-1} V F^{-1}=\Sigma$, i.e., $V=F^{\prime} \Sigma F$. By the definition of $F$, the $(i, j)$ element of $F^{\prime} \Sigma F$ is equal to

$$
\left(\frac{-1}{\delta}\right)^{i+j} \sum_{m=1}^{i} \sum_{n=1}^{j}(-1)^{m+n}\left(\begin{array}{c}
i \\
m
\end{array}\right)\left(\begin{array}{l}
j \\
n
\end{array}\right) \sigma_{m n} .
$$

Since $\sigma_{m n}=E_{\theta}\left\{f(X, \theta+m \delta) f(X, \theta+n \delta) / f^{2}(X, \theta)\right\}-1$, it follows $\sigma_{m 0}=$ $\sigma_{0 n}=0$ for all $m$ and $n$. Thus (2.5) is equal to

$$
\begin{aligned}
& \left(\frac{-1}{\delta}\right)^{i+j} \sum_{m=0}^{i} \sum_{n=0}^{j}(-1)^{m+n}\left(\begin{array}{c}
i \\
m
\end{array}\right)\left(\begin{array}{l}
j \\
n
\end{array}\right)\left[E_{\theta}\left\{\frac{f(X, \theta+m \delta) f(X, \theta+n \delta)}{f^{2}(X, \theta)}\right\}-1\right] \\
= & \left(\frac{-1}{\delta}\right)^{i+j} \sum_{m=0}^{i} \sum_{n=0}^{j}(-1)^{m+n}\left(\begin{array}{c}
i \\
m
\end{array}\right)\left(\begin{array}{l}
j \\
n
\end{array}\right) E_{\theta}\left\{\frac{f(X, \theta+m \delta) f(X, \theta+n \delta)}{f^{2}(X, \theta)}\right\} \\
= & E_{\theta}\left(\Psi_{i} \Psi_{j}\right),
\end{aligned}
$$

by using $\sum_{n=0}^{j}(-1)^{n}\left(\begin{array}{l}j \\ n\end{array}\right)=(1-1)^{j}=0$. This is the $(i, j)$ element of $V$. Thus we complete the proof. 
Assume the regularity conditions $R_{k}$ which the Bhattacharyya bound (1.1) holds, that is, $S(\theta)$ is independent of $\theta$ and $f(x, \theta)$ is sufficiently smooth that $k$-th derivatives with respect to $\theta$ of the left-hand sides of

$$
\int_{S(\theta)} f(x, \theta) d \mu=1
$$

and

$$
\int_{S(\theta)} \hat{g}(x) f(x, \theta) d \mu=g(\theta)
$$

can be obtained by differentiating $k$ times under the integral signs. Then we have the following corollary.

Corollary. Under the regularity conditions $R_{k}$, it holds

$$
B_{k}(\theta) \leq D_{k}(\theta) \leq K_{k}(\theta) \quad(k \geq 1)
$$

Proof. At first we will show the left inequality. We have from Lemma 1 that, for $i=1, \ldots, k$,

$$
\begin{aligned}
G_{i} & \rightarrow g^{(i)}(\theta) \\
\Psi_{i} & =\left(\frac{-1}{\delta}\right)^{i} \sum_{l=0}^{i}\left(\begin{array}{l}
i \\
l
\end{array}\right)(-1)^{l} \frac{f(x, \theta+l \delta)}{f(x, \theta)} \rightarrow \frac{\partial^{i} f(x, \theta) / \partial \theta^{i}}{f(x, \theta)}
\end{aligned}
$$

as $\delta \rightarrow 0$. Then we have

$$
\begin{aligned}
\lim _{\delta \rightarrow 0} v_{i j}(\theta, \delta) & =\lim _{\delta \rightarrow 0} E_{\theta}\left(\Psi_{i} \Psi_{j}\right)=E_{\theta}\left(\lim _{\delta \rightarrow 0} \Psi_{i} \Psi_{j}\right) \\
& =E_{\theta}\left\{\frac{\partial^{i} f(X, \theta) / \partial \theta^{i}}{f(X, \theta)} \frac{\partial^{j} f(X, \theta) / \partial \theta^{j}}{f(X, \theta)}\right\} .
\end{aligned}
$$

So, taking the limit of the right-hand side of (2.4),

$$
\begin{aligned}
\lim _{\delta \rightarrow 0} \mathbf{g} V^{-1} \mathbf{g}^{\prime} & =\lim _{\delta \rightarrow 0}\left(G_{1}, \ldots, G_{k}\right) V^{-1}\left(G_{1}, \ldots, G_{k}\right)^{\prime} \\
& =\left(g^{(1)}(\theta), \ldots, g^{(k)}(\theta)\right) W^{-1}(\theta)\left(g^{(1)}(\theta), \ldots, g^{(k)}(\theta)\right)^{\prime}
\end{aligned}
$$

where $W=W(\theta)=\left\{w_{i j}(\theta)\right\}$ with

$$
w_{i j}(\theta):=E_{\theta}\left[\frac{\frac{\partial^{i}}{\partial \theta^{i}} f(X, \theta)}{f(X, \theta)} \cdot \frac{\frac{\partial^{j}}{\partial \theta^{j}} f(X, \theta)}{f(X, \theta)}\right] \quad(i, j=1, \ldots, k) .
$$


Hence

$$
\begin{aligned}
& \lim _{\delta \rightarrow 0} \mathbf{g} V^{-1} \mathbf{g}^{\prime} \\
= & \left(g^{(1)}(\theta), \ldots, g^{(k)}(\theta)\right) W^{-1}(\theta)\left(g^{(1)}(\theta), \ldots, g^{(k)}(\theta)\right)^{\prime} \\
\leq & \sup _{\delta \in \Delta} \mathbf{g} V^{-1} \mathbf{g}^{\prime},
\end{aligned}
$$

the bound (2.3) is as great as the Bhattacharyya bound at least.

For the right inequality, we may apply the Theorem 2. Thus we complete the proof.

\section{EXAMPLES}

In this section, we show some examples.

Example 1. Unbiased estimation of the standard deviation of a normal distribution. Let $X_{1}, X_{2}$ be independent, identically distributed random variables according to the normal distribution with mean 0 and variance $\theta^{2}(\theta>0)$. Then the joint density of $\left(X_{1}, X_{2}\right)$ is

$$
f(x, y, \theta)=\left(2 \pi \theta^{2}\right)^{-1} \exp \left\{-\left(x^{2}+y^{2}\right) /\left(2 \theta^{2}\right)\right\}
$$

Since $s^{2}=\left(X_{1}^{2}+X_{2}^{2}\right) / 2$ is a complete sufficient statistic for $\theta, \hat{g}\left(X_{1}, X_{2}\right)=$ $2 s / \sqrt{\pi}$ is the uniformly minimum variance unbiased (UMVU) estimator of $\theta$ with the variance $\{(4 / \pi)-1\} \theta^{2} \approx 0.2732 \theta^{2}$. By a straightforward computation, it follows that $B_{1}(\theta)=0.25 \theta^{2}$ and $B_{2}(\theta)=17 \theta^{2} / 64 \approx 0.2656 \theta^{2}$. In Kshirsagar (2000), the value of $K_{k}(\theta)$ is calculated by taking $\phi_{i}=\theta+i \delta(i=1, \ldots, k)$. In this case, since $\mathbf{g} V^{-1} \mathbf{g}^{\prime}$ in (2.3) is equal to $\mathbf{w} \Sigma^{-1} \mathbf{w}^{\prime}$ in (1.2) from Theorem 2, we obtain the same numerical values in Table 1 in Kshirsagar (2000) (see also Sen and Ghosh (1976)). For example, we have $H(\theta)=K_{1}(\theta)=D_{1}(\theta) \approx$ $0.2698 \theta^{2}>B_{2}(\theta)$.

Example 2. Unbiased estimation of the scale of uniform distribution. Let $X$ be a random variable with the uniform distribution over $(0, \theta)$. (i) If $g(\theta)=\theta$, since $X$ is a complete sufficient statistic for $\theta$, then $\hat{g}(X)=2 X$ is the UMVU 
estimator of $g(\theta)$ with the variance $\theta^{2} / 3 \approx 0.333 \theta^{2}$. We have for the bound (1.2)

$$
\begin{aligned}
& \frac{\{g(\theta+\delta)-g(\theta)\}^{2}}{E_{\theta}\left\{\frac{f(X, \theta+\delta)-f(X, \theta)}{f(X, \theta)}\right\}^{2}}=-\delta(\theta+\delta) \quad(-\theta<\delta<0), \\
& H(\theta)=K_{1}(\theta)=\sup _{\delta} \frac{\{g(\theta+\delta)-g(\theta)\}^{2}}{E_{\theta}\left\{\frac{f(X, \theta+\delta)-f(X, \theta)}{f(X, \theta)}\right\}^{2}}=\frac{\theta^{2}}{4}=0.25 \theta^{2} .
\end{aligned}
$$

Putting $\phi_{i}=\theta+\delta_{i}\left(i=1,2, \delta_{1} \neq \delta_{2}\right)$ in (1.3), we have, for $k=2$,

$$
\begin{aligned}
& \mathbf{w} \Sigma^{-1} \mathbf{w}^{\prime}=\frac{1}{\theta} \delta_{1}\left(\theta+\delta_{2}\right)\left(\theta+\delta_{1}-\delta_{2}\right) \quad\left(-\theta<\delta_{2}<\delta_{1}<0\right), \\
& K_{2}(\theta)=\sup _{\phi} \mathbf{w} \Sigma^{-1} \mathbf{w}^{\prime}=\frac{8}{27} \theta^{3} \approx 0.296 \theta^{3} .
\end{aligned}
$$

Moreover, we have for the bound (2.3)

$$
\begin{aligned}
\mathbf{g} V^{-1} \mathbf{g}^{\prime} & =\frac{-2 \delta(\theta+\delta)^{2}}{\theta} \quad(-\theta / 2<\delta<0), \\
\sup _{\delta} \mathbf{g} V^{-1} \mathbf{g}^{\prime} & =\frac{8}{27} \theta^{3} \approx 0.296 \theta^{3},
\end{aligned}
$$

thus $K_{2}(\theta)=D_{2}(\theta)>H(\theta)$ for all $\theta \in \Theta$ in this case. (ii) If $g(\theta)=\theta^{2}$, then $\hat{g}(X)=3 X^{2}$ is the UMVU estimator with the variance $0.8 \theta^{4}$. We have for the bound (1.2)

$$
\begin{aligned}
& \frac{\{g(\theta+\delta)-g(\theta)\}^{2}}{E_{\theta}\left\{\frac{f(X, \theta+\delta)-f(X, \theta)}{f(X, \theta)}\right\}^{2}}=-(2 \theta+\delta)^{2}(\theta+\delta) \delta \quad(-\theta<\delta<0), \\
H(\theta)=K_{1}(\theta)= & \sup _{\delta} \frac{\{g(\theta+\delta)-g(\theta)\}^{2}}{E_{\theta}\left\{\frac{f(X, \theta+\delta)-f(X, \theta)}{f(X, \theta)}\right\}^{2}} \\
= & \frac{-\theta^{4}}{4096}(9+\sqrt{17})^{2}(1+\sqrt{17})(-7+\sqrt{17}) \approx 0.620 \theta^{4} .
\end{aligned}
$$

Putting $\phi_{i}=\theta+\delta_{i}\left(i=1,2, \delta_{1} \neq \delta_{2}\right)$ in (1.3), we have, for $k=2$,

$$
\begin{aligned}
& \mathbf{w} \Sigma^{-1} \mathbf{w}^{\prime} \\
&=\frac{1}{\theta} \delta_{1}\left(\theta-\delta_{2}\right)\left(-\delta_{1}^{3}-\delta_{1}^{2}-\delta_{1}^{2} \delta_{2}+\delta_{1} \delta_{2}^{2}+\delta_{1} \delta_{2} \theta+\delta_{1} \theta^{2}+\theta^{3}+\delta_{2}^{3}+\delta_{2}^{2} \theta+\theta^{2} \delta_{2}\right) \\
& \quad\left(-\theta<\delta_{2}<\delta_{1}<0\right),
\end{aligned}
$$




$$
\begin{aligned}
K_{2}(\theta)= & \sup _{-\theta<\delta_{2}<\delta_{1}<0} \mathbf{w} \Sigma^{-1} \mathbf{w}^{\prime}=\frac{-\theta^{4}}{13107200000}(23+\sqrt{17}+\sqrt{7906+366 \sqrt{17}}) \\
& \cdot(-1240+24 \sqrt{17}+\sqrt{7906+366 \sqrt{17}}+\sqrt{17} \sqrt{7906+366 \sqrt{17}}) \\
& \cdot(277 \sqrt{7906+366 \sqrt{17}}+51 \sqrt{17} \sqrt{7906+366 \sqrt{17}} \\
& +57958+3210 \sqrt{17}) \\
\approx 0.723 \theta^{4} &
\end{aligned}
$$

Moreover, we have for the bound (2.3)

$$
\begin{aligned}
\mathbf{g} V^{-1} \mathbf{g}^{\prime}= & \frac{-2 \delta(\theta+\delta)^{2}\left(9 \delta^{2}+8 \theta \delta+4 \delta^{2}\right)}{\theta} \quad(-\theta / 2<\delta<0) \\
\sup _{\delta} \mathbf{g} V^{-1} \mathbf{g}^{\prime} & \\
=\frac{-2 \theta^{4}}{1660} & {\left[\left\{53125[19801+270 \sqrt{5745}\}^{2 / 3}-299\right.\right.} \\
& \left.-59\{19801+270 \sqrt{5745}\}^{1 / 3}\right] \\
& \cdot\left[76\{19801+270 \sqrt{5745}\}^{1 / 3}+\{19801+270 \sqrt{5745}\}^{2 / 3}-299\right]^{2} \\
& \cdot\left[6401\{19801+270 \sqrt{5745}\}^{1 / 3}\right. \\
& +90\{19801+270 \sqrt{5745}\}^{1 / 3} \sqrt{5745} \\
& \left.+1301\{19801+270 \sqrt{5745}\}^{2 / 3}+43001+180 \sqrt{5745}\right] \\
& \cdot\left\{19801+270 \sqrt{5745}^{5 / 3}\right\}^{-1} \\
\approx 0 . & 21 \theta^{4},
\end{aligned}
$$

thus $K_{2}(\theta)>D_{2}(\theta)>H(\theta)$ for all $\theta \in \Theta$ in this case.

\section{ACKNOWLEDGEMENTS}

The author would like to thank Professor M. Akahira for his encouragement throughout the preparation of the paper. 


\section{REFERENCES}

Bhattacharyya, A. (1946). On some analogues of the amount of information and their uses in statistical estimation. Sankhya 8, 1-14, 201-218, 315-328.

Chapman, D. G. and Robbins, H. (1951). Minimum variance estimation without regularity assumptions. Ann. Math. Statist., 22, 581-586.

Fend, A. V. (1959). On the attainment of Cramér-Rao and Bhattacharyya bounds for the variance of an estimate. Ann. Math. Statist., 30, 381-388.

Hammersley, J. M. (1950). On estimating restricted parameters. J. Roy. Statist. Soc. Ser. B, 12, 192-240.

Kiefer, J. (1952). On minimum variance estimators. Ann. Math. Statist., 23, $627-629$.

Kshirsagar, A. M. (2000). An extension of the Chapman-Robbins inequality. J. Indian Statist. Assoc., 38, 355-362.

Sen, P. K. and Ghosh, B. K. (1976). Comparison of some bounds in estimation theory. Ann. Statist., 4, 755-765. 